\title{
VELOCITY AND SURFACE-LOWERING MEASUREMENTS ON LAW DOME, ANTARCTICA \\ (Abstract)
}

by

N. Young, P. Malcolm, P. Mantell,

(Australian Antarctic Division, Channel Highway, Kingston, Tasmania 7050, Australia)

and

E. McGibbon

(Australian Survey and Land Information Group, P.O. Box 2, Belconnen, ACT 2616, Australia)

Law Dome is a medium-sized ice cap, about $200 \mathrm{~km}$ in diameter, adjoining the main Antarctic ice sheet in Wilkes Land. Surveys of ice thickness, velocity, and accumulation on a triangular network were first made in 1965 and 1966. Analysis of the data indicated that the surface elevation along a major sector of a line from the summit of Law Dome to the north coast is lowering at a rate between 0.4 and $0.8 \mathrm{~m} \mathrm{a}^{-1}$ (Pfitzner, 1980). In 1987, surveys were made over the same north-south line in an attempt to detect any change in the surface elevation, which could be up to $16 \mathrm{~m}$ lower over the approximately 20 year interval. Two techniques were employed: direct comparison of measured surface elevations, and implied surface-elevation change by comparison of gravity measurements at the same geographic location.

Surface elevations were measured by a closed optical levelling traverse in 1968. Six of the original survey sites were accurately re-located in 1987 and the elevation of the snow surface was determined using doppler-satellite surveys. Elevation determined by optical levelling is referenced to mean sea-level and that determined by satellite surveys to an Earth-centred reference system. Conversion of data between the two systems requires a knowledge of the local geoidal undulations. Preliminary analysis of the data from the satellite surveys, after making an approximate adjustment for the local geoid, indicates that any possible change in surface elevation is less than $10 \mathrm{~m}$ and probably less than $5 \mathrm{~m}$.
The value of gravity at a given geographic location on the ice cap will increase with decreasing elevation, and decrease with decreasing ice thickness. For a decrease in surface elevation due to a change in ice thickness, the gravity will increase at a rate of about $1 \mu \mathrm{m} \mathrm{s}^{-2} / 0.5 \mathrm{~m}$ elevation decrease. Preliminary analysis of the gravity data suggests that the elevation change is less than $3-5 \mathrm{~m}$ along the line and probably close to zero.

In calculating the surface-lowering rate at a point, Pfitzner (1980) made assumptions about the distribution of strain-rate in the ice sheet. $\mathrm{He}$ used the measured strain-rates along the survey line and inferred strain-rates transverse to the survey line from the broad-scale distribution of velocity and strain-rate measured around the triangular network. In addition, he assumed the strain-rates averaged through the ice thickness are about $90 \%$ of the surface values. Errors in these assumptions and in the measured distributions will make significant contributions to the calculated lowering rate. Additional measurements of velocity, ice thickness, surface elevation, and accumulation rate were made in 1987 at points spread over Law Dome in order to resolve the discrepancy between the predicted and measured lowering rates.

\section{REFERENCE}

Pfitzner, M.L. 1980. The Wilkes Ice Cap Project., 1966. ANARE Sci. Rep., Ser. A(4), glaciol. (Publ. 127.) 